\title{
A possible mechanism for the formation of annual growth lines in bivalve shells
}

\author{
LIU XiaoJun ${ }^{1}$, YAN ZhenGuang ${ }^{3}$, ZHENG GuiLan ${ }^{1}$, ZHANG GuiYou ${ }^{1}$, \\ WANG HongZhong ${ }^{1}$, XIE LiPing ${ }^{1,2^{*}}$ \& ZHANG RongQing $^{1,2^{*}}$ \\ ${ }^{1}$ Institute of Marine Biotechnology, School of Life Sciences, Tsinghua University, Beijing 100084, China; \\ ${ }^{2}$ Protein Science Laboratory of the Ministry of Education, Tsinghua University, Beijing 100084, China; \\ ${ }^{3}$ Key Lab of Ecological Effect and Risk Assessment for Chemicals, Chinese Research Academy of \\ Environmental Sciences, Beijing 100012, China
}

Received March 20, 2009; accepted June 28, 2009

\begin{abstract}
We report a unique shell margin that differed from the usual shell structure of Pinctada fucata. We observed empty organic envelopes in the prismatic layer and the formation of the nacreous layer in the shell margin. All the characteristics of the growing margin indicated that the shell was growing rapidly. To explain this anomaly, we propose the concept of "jumping development". During jumping development, the center of growth in the bivalve shell jumps forward over a short time interval when the position of the mantle changes. Jumping development explains the unusual structure of the anomalous shell and the development of annual growth lines in typical shells. Annual growth lines are the result of a discontinuity in the shell microstructure induced by jumping development.
\end{abstract}

bivalves, shell microstructure, internal growth bands, the prismatic layer, the nacreous layer

Citation: Liu X J, Yan Z G, Zheng G L, et al. A possible mechanism for the formation of annual growth lines in bivalve shells . Sci China Life Sci, 2011, 54: 175-180, doi: 10.1007/s11427-010-4132-z

Bivalves produce annual growth lines in the cross-section of their shells that closely resemble the annual rings in trees and the annual growth bands in corals [1-3]. The annual growth increment is typically measured as the height of the shell between two adjacent lines [4,5]. These lines can be used to reliably determine the age of an individual bivalve [6-8]. The variation in shell growth rates between different years is indicative of environment changes [9]. For example, shell growth rates vary with changes in temperature and food supply $[2,10]$. These traits have proven useful in ecological, geochemical, and paleontological studies for assessing the effects of a range of biological, anthropogenic, and environmental stressors [4,11-15].

Bivalves are an ideal indicator of environmental changes

*Corresponding author (email: lpxie@mail.tsinghua.edu.cn; rqzhang@mail.tsinghua edu.cn) for several reasons. The majority of species are long-lived which allows the study of environmental changes over a long period of time [16]. Furthermore, bivalves are widely distributed, inhabiting fresh water, coastal waters, the deep sea, and several habitats that are characterized by extreme environmental conditions $[11,17,18]$. The growth patterns of bivalve shells can be used to study both recent and historic changes in local and seasonal climates [19]. Moreover, differences in the pattern of growth in a single species can be used to study the adaptation of organisms to different environmental conditions. Last, 20000 species of marine bivalves have been documented to date, representing a rich diversity of life histories. However, the impact of environmental factors on the natural population of bivalves can only be studied once the annual growth patterns are calibrated for a given population $[8,20,21]$. 
Although shell growth rates are influenced by environment factors, a one-to-one relationship between a given factor and a given rate has not been established. This is partly because the mechanism of the formation of annual growth lines is poorly understood. Based on our analysis of a unique growth band discovered in the shell margin of a Pinctada fucata individual, we propose the concept of "jumping development" in bivalve shells to explain the formation of annual growth lines.

\section{Materials and methods}

We obtained pearl oyster, $P$. fucata, individuals from the Guofa Pearl Farm in Beihai, Guangxi Province, China. A single shell, collected in October 2007, contained a unique growth margin. This shell is hereafter referred to as the anomalous shell. The remaining 20 shells used in this study did not contain this unique margin and are therefore referred to as "typical" shells. The shells were $\sim 4.5-6.0 \mathrm{~cm}$ in diameter and 32-40 g in wet weight. The shells were cleaned and air dried, then the regions of the shell containing only the prismatic layer were separated from the regions containing both the prismatic layer and the nacreous layer. The tissue containing only the prismatic layer was coated with gold and observed using scanning electron microscopy (SEM). We compared SEM images of the margin of the anomalous shell with those of the typical shells. In addition, we compared the morphology of the different regions of the unique growth band with the previously reported microstructure of other shells. We used the Raman spectra to identify the nature of the crystals in the unique growth band. The Raman spectra were recorded at an excitation wavelength of $514 \mathrm{~nm}$, provided by an argon laser (limited to 4.6 $\mathrm{mW})$.

\section{Results}

The anomalous shell exhibited a unique margin on the inner surface of the shell (Figure 1A and B), outside the region containing the mature prismatic layer. The widest part of this band was $1 \mathrm{~mm}$. The newly forming prismatic layer in this unique margin possessed the same characteristics as the mature prismatic layers of other taxa. Each prism was surrounded by an interprismatic organic envelope (Figure 1B). In addition, small empty organic cavities and large developing organic cavities were visible in the recesses of the margin (Figure 1A and B, white arrows). The surface of the growing prism was raised and exhibited a concentric pattern, which is distinctly different from the flat surface of a mature prism (Figure 2A).

The unique band contained laminate-like structures (Figure 1A). The Raman analysis revealed a sharp peak at 1085 $\mathrm{cm}^{-1}$, confirming that the crystals in these structures were aragonites (Figure 1F). The morphology indicates that they constitute the nacreous layer (Figure 1D). The nacre growth in the unique band differed from the generic growth observed in the nacreous layer of other shells. The nacreous layer in the unique margin (Figure 1D) was similar in shape to the fractured nacreous layer. Compared with the fractured nacreous layer, the hierarchy between layers is clear and the lines between layers are smooth [22].

The unique margin contained two organic layers separated by aragonite crystals. The first organic layer covered the aragonites (Figure 1A). The organic layer between the exposed growth band and the mature prismatic layer (Figure 2A) constituted the inner surface of the shell. The transition between the organic layer and mature prismatic layer was visible $\sim 8 \mathrm{~mm}$ from the shell margin (Figure $2 \mathrm{~B}$ ) illustrating the formation of the organic layer. The organic envelope surrounding the prism is formed by the addition of an organic matrix to its surface (Figure $2 \mathrm{C}$ ). The organic matrix is continuously added and grows toward the center of the prisms' surface (Figure 2D) until the surface is completely covered by the organic matrix. The trail of the raised organic envelope is ultimately invisible (Figure $2 \mathrm{E}$ ). The second organic layer consists of an organic interlayer that covers the earlier formed prismatic layer. Aragonites grow quickly on this layer (Figure 1B and E).

In a typical $P$. fucata shell, the edge consists of an outer periostracum that is not visible on a SEM map and an inner prismatic layer, both of which are mature structures (Figure 1C). We did not observe the unique band in any of the 20 typical shells.

\section{Discussion}

\subsection{The difference in microstructure between the unique band and typical shells}

The unique margin of the anomalous shell consists of five layers. These include, in order from the outer to the inner layer, an outer periostracum, a growing prismatic layer, an organic nacre-prism interlayer, a growing nacreous layer, and an organic layer covering the nacreous layer (Figure 1A). Together, these layers constitute the growth front of the shell. Given this, it is interesting that so many of these layers differ in structure from those of the typical shells.

Historically, it was thought that the interprismatic organic membrane of the prism was created by squeezing between the prisms during their formation [23,24]. However, Checa et al. [25] proposed that calcite grows in organic cavities that are formed at an earlier stage. Although organic macromolecules are believed to self-assemble into regular structures in vitro during the process of shell biomineralization, empty organic envelopes cannot always be observed in the prismatic layer in typical shells (Figure 1C), as was the 

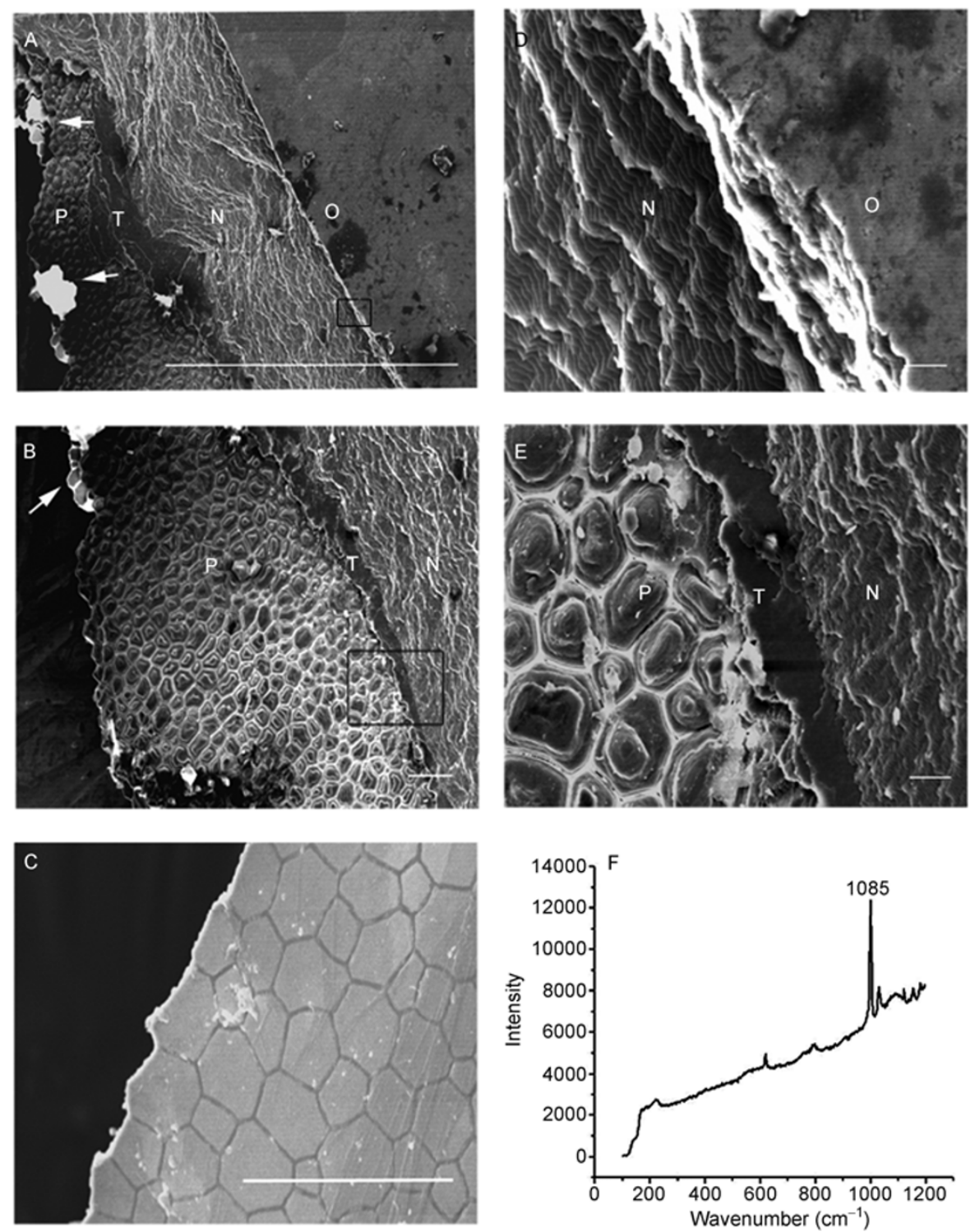

Figure 1 SEM images of the shell margin of a special Pinctada fucata shell and a typical $P$. fucata shell. P, the calcite prismatic layer; T, the prism-nacre transition; N, the aragonite nacreous layer; $\mathrm{O}$, the organic layer covering the newly formed nacreous layer. A, The unique growing shell margin in the anomalous shell. The upgrowth sequence of every layer is in accordance with the development of the general shell. Scale bar=1 mm. B, The broadest part of the unique growing shell margin in the anomalous shell. Scale bar=100 $\mu \mathrm{m}$. C, The mature prismatic layer in the margin of a typical shell. Scale bar=100 $\mu \mathrm{m}$. D, Enlarged image of the boxed region in A. Scale bar $=10 \mu \mathrm{m}$. E, Enlarged image of the boxed region in B. Scale bar=20 $\mu \mathrm{m}$. F, Raman spectrum analysis of the crystal in D. The direction of growth is from right to left in the shell.

case in the unique margin in this study. We propose that the location of the empty cavities indicates that they are not the result of seawater cauterization. Thus, the organic envelope formed prior to the biomineralization of the calcite prisms. We hypothesize that the mantle first secretes the periostracum then secretes organic macromolecules onto the periostracum to develop the organic cavities. Organic macromolecules form a wall-like envelope on the periostracum and separate this envelope into small organic cavities.

The most unusual feature of the margin in the anomalous shell is that the nacreous layer occurs in the shell margin. Moreover, the pattern of growth of the nacre in the growth band was unusual (Figure 3A). Growth did not resemble simple ladder-like growth (Figure 3B), the typical pattern of a generic nacreous layer [26], and some layers appeared to shrink regularly (Figure 1D). We named this unique pattern "complex ladder-like growth". When the height of the two kinds of nacreous layer is equal $\left(h_{1}=h_{2}\right)$, the breadth of the growth surface is far narrower than that seen in simple ladder-like growth $\left(b_{1}<<b_{2}\right.$, Figure $3 \mathrm{~A}$ and $\left.\mathrm{B}\right)$. Complex ladder-like growth results in a more stable and harder nacreous layer. We propose that it also can lead to a step-growth pattern of the nacre at the inner surface of the shell (Figure 3C). The first organic layer found in the anomalous shell margin 

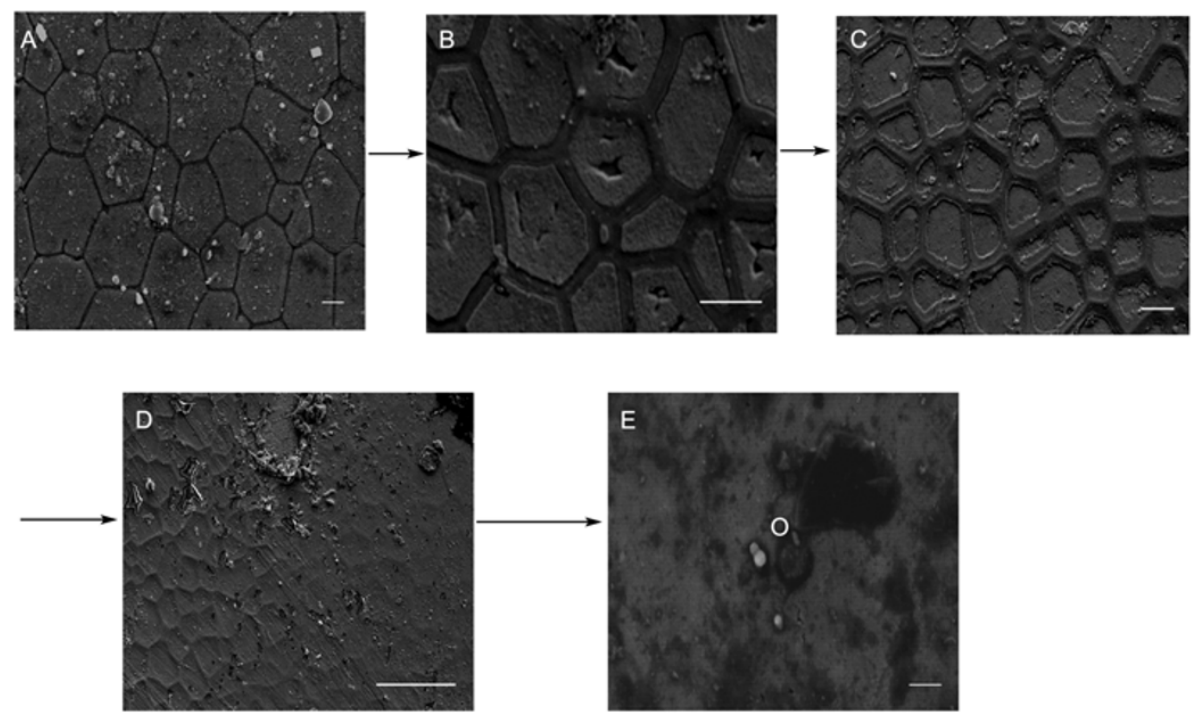

Figure 2 SEM images of the formation of the organic layer covering the nacreous layer in the unique growing shell margin. O, the organic layer covering the newly formed nacreous layer. A, The mature prismatic layer $\sim 8 \mathrm{~mm}$ from the special growing shell margin. B-D, The transition of the mature prismatic layer and the organic layer covering the nacreous layer. E, The organic layer covering the nacreous layer. Scale bar=20 $\mu \mathrm{m}$ in A-C; scale bar=100 $\mu \mathrm{m}$ in D; scale bar $=10 \mu \mathrm{m}$ in $\mathrm{E}$. The direction of growth is from left to right in the shell.

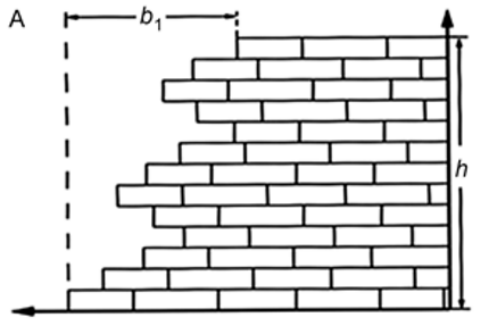

Complex ladder-like growth

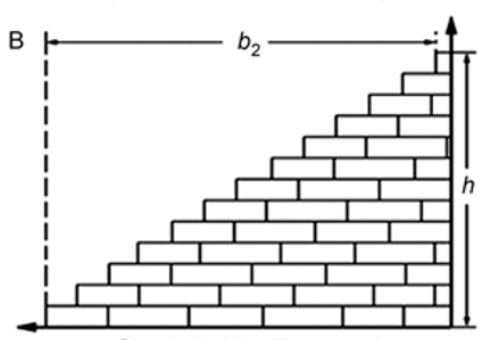

Simple ladder-like growth

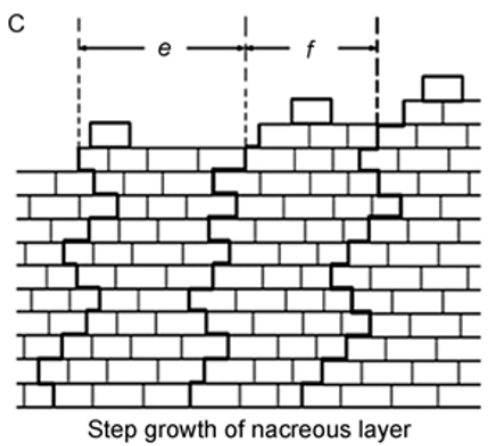

Figure 3 Models of growth in the nacreous layer of the growth band in the anomalous shell and typical shells. A, The complex ladder-like growth of the nacreous layer in the unique band of the anomalous shell. B, The simple ladder-like growth of the nacreous layer in a typical shell. C, The step growth of the nacreous layer in the unique band of the anomalous shell. was not found in other areas in the shell or in other typical $P$. fucata shells. Thus, it is a unique structure that was formed to protect the newly formed nacreous layer. An interesting characteristic of this layer is that its direction of growth is opposite the direction of shell growth. The organic interlayer between the prismatic layer and nacreous layer is similar to the prism-nacre transition layer in a typical shell, and the layers have the same action. The nacreous layer grows on the organic interlayer. Thus, we believe that it is a natural structure in the shell, although it differs from the prism-nacre transition in appearance.

All of the characteristics of the anomalous shell margin suggest that it was growing rapidly. In the newly forming prismatic layer, the prisms appear to have been recently formed. Calcites grow quickly immediately after the formation of organic envelopes and the surface of the prismatic layer was covered by the organic interlayer (Figure 1E) before it grew to form the smooth mature prismatic layer (Figure 1C). The organic interlayer provides the surface for the first nacre nucleation. However, we were unable to observe the nucleation of the nacre. In addition, we did not observe the growth of the aragonite plates to their normal size. The complex ladder-like growth pattern results in a stable nacreous layer and also shortens the growth time. Thus, growth expands rapidly and the new nacreous layer grows over the first organic layer when the growth of the unique band is complete.

\subsection{Mechanism for the formation of annual growth lines in bivalve shells}

This rapid growth could account for the observed differences between the layers in typical shells and those in the 
unique growing shell margin. However, the following questions remain: Why did the nacreous layer occur in the shell margin and why did the special growing shell margin form? The mantle secretes matrix macromolecules to control the growth of the shell, thus any change in the mantle affects shell growth. Different zones of the mantle play different roles in shell growth [27]. Biomacromolecules secreted by the central zone of the mantle construct the nacreous layer and those secreted from the edge of the mantle construct the prismatic layer. Thus, when the position of the mantle changes relative to the shell, shell growth will become disordered. It does not seem possible for the position of the mantle relative to the shell to change in nature. However, it is interesting that the nacreous layer formed outside the mature prismatic layer in the growth band. If the relative position is unchangeable, the nacreous layer will never occur in the margin of the shell. Thus, the most probable explanation for the formation of the unique growing shell margin is that a change in the pattern of growth occurred in the anomalous shell, which in turn changed the position of the mantle.

We propose the following scenario for the development of the unique growing shell margin and of annual growth lines. We hypothesize that the speed of growth of the shell does not always match the speed of growth of the mantle. Cell multiplication in the central zone of the mantle is more rapid than that in the marginal zone of the mantle [28] and the speed of growth is faster in bivalves cultured in high temperatures than at low temperatures. When the central zone of the mantle grows rapidly in high temperatures and the shell cannot keep up, the mantle will pucker inside the shell (Figure 4A and B). Thus, a change in the growth pattern of the shell will occur before the shell growth and mantle growth resynchronize (Figure $4 \mathrm{C}$ and $\mathrm{D}$ ). When the mantle puckers, it spreads so that the edge of the mantle moves from the normal position and the area of the mantle that secretes matrix macromolecules to form the nacreous layer covers the prismatic layer. Because the growth of the shell in this manner is discontinuous and paroxysmal, we termed it "jumping development". When the mantle puckers and the position of the mantle changes, the normal pattern of growth is disrupted. Mantle secretion does not stop, but the matrix macromolecules cannot accurately direct biomineralization in either the prismatic layer or the nacreous layer. The new prismatic layer forms first on the margin of the shell. Part of the central zone of the mantle will cover the mature prismatic layer. Thus, a belt of the nacreous layer forms on the new prismatic layer at the shell edge because the matrix macromolecules secreted by this area of mantle direct formation of the nacreous layer. The unique growing shell margin is formed during this process (Figure 4D). Moreover, the growth of the nacreous layer and prismatic layer in other areas will not be continuous. Thus, the prism-nacre transition disappears and new nacreous layers form directly on the surface of prismatic layers and the old nacreous layer. This state remains until the growth of the mantle once again corresponds to the growth of the shell. These processes create the discontinuity in the microstructures of the prismatic and nacreous layers. The discontinuity of the two layers results in the formation of annual growth lines in the cross section of shells. Thus, the surface of the old prismatic layer and nacreous layer represents the location of the annual growth line (Figure 4E). Because the growth surface of the mineralized structure is changed, the macromolecules that have been deposited cannot direct the biomineralization process continuously until shell growth becomes order-continuous again.

The content of organic matrix in the area of annual growth lines is higher than that in other areas [29]. The formation of the organic layer covering the nacreous layer in the unique growing shell margin can be explained by this phenomenon. The ultimate fate of the deposited organic matrix is represented by the organic layer covering the special growing shell margin (Figure 2E). When the secretion of organic macromolecules proceeds, it covers the original prismatic layer entirely and disrupts the pattern of growth in the old nacreous layer. Thus, the jumping development of $P$. fucata represents paroxysmal growth of the shell that results from the disaccord between the growth of the mantle and the shell. Different rates of cell proliferation during different seasons and in different zones of the mantle explain the

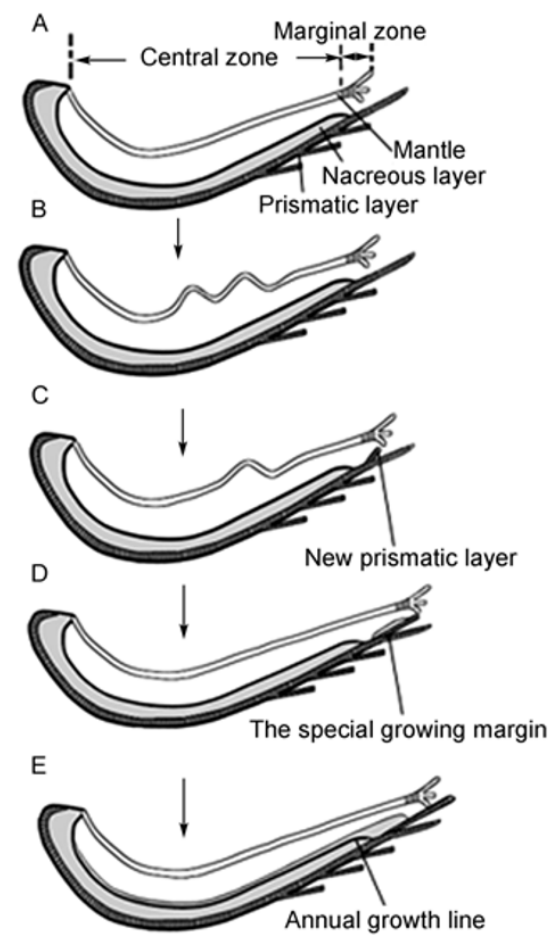

Figure 4 Jumping development and the formation of annual growth lines. A, The mantle and shell of P. fucata. B, The mantle puckers because of mantle overgrowth. C, A new prismatic layer forms when the mantle moves forward because of the power generated by the pucker. D, The unique growing shell margin forms when part of the central zone, which secretes organic macromolecules that control nacre formation, covers the prismatic layer. E, Annual growth line forms when the new nacre covers the unique growing shell margin and nacreous layer. 
disaccord. Annual growth lines result from the discontinuity in the microstructures of shells induced by jumping development.

Our hypotheses advance a relationship between the mantle and annual patterns in the shell. Attempts to understand forcing and feedbacks within the aquatic ecosystem require that the effect of environmental factors such as temperature and food supply [9] on the growth rates of shells in different years be measurable. Past studies of annual growth lines have focused on changes in shell microstructure. However, the instantaneous changes in microstructure that occur are difficult to measure in real time because correlative indexes of microstructures do not exist. However, indexes of bivalve mantles, such as gene expression, protein secretion, enzyme activity, and cell multiplication may be used to characterize the changes that occur in the mantle. Norkko et al. [17] used nucleic acid ratios as a measure of protein synthesis potential and examined the possibility of using this ratio to assess short-term growth rates in a range of marine organisms. By studying the characteristics of the mantle and variations in these characteristics that occur over space and time, a one-to-one correspondence between environmental factors and bivalve mantles may be established. Such indexes for bivalve mantles also represent the factors that influence shell formation. Therefore, correlations between environmental factors and annual growth rates of shells throughout a bivalve's lifetime can be detected and measured within real time.

This work was supported by the National Natural Science Foundation of China (Grant Nos. U0831001 and 40876068) and the National High Technology Research and Development Program of China (Grant Nos. 2010CB126405 and 2010AA09Z405). We are grateful to Mr. Lynn Lauerman for help with manuscript preparation.

1 Emiliani C, Hudson J H, Shinn J A, et al. Oxygen and carbon isotopic growth record in a reef coral from the Florida Keys and a deep-sea coral from Blake Plateau. Science, 1978, 202: 627-629

2 Jones D S. Annual growth increments in shells of Spisula solidissima record marine temperature variability. Science, 1981, 211: 165-167

3 Abele D, Brey T, Philipp E. Bivalve models of aging and the determination of molluscan lifespans. Exp Gerontol, 2009, 44: 307-315

4 Jones D S. Annual cycle of shell growth increment formation in two continental shelf bivalves and its paleoecologic significance. Paleobiology, 1980, 6: 331-340

5 Rhoads D C, Lutz R A, Revelas E C, et al. Growth of bivalves at deep-sea hydrothermal vents along the Galápagos Rift. Science, 1981, 214: 911-913

6 Arneri E, Giannetti G, Antolini B. Age determination and growth of Venus verrucosa L. (Bivalvia: Veneridae) in the southern Adriatic and the Aegean Sea. Fish Res, 1998, 38: 193-198

7 Sejr M K, Jensen K T, Rysgaard S. Annual growth bands in the bivalve Hiatella arctica validated by a mark-recapture study in NE Greenland. Polar Biol, 2002, 25: 794-796

8 Moura P, Gaspar M B, Monteiro C C. Age determination and growth rate of a Callista chione population from the southwestern coast of Portugal. Aquat Bio, 2009, 5: 97-106

9 Richardson C A. Molluses as archives of environmental change.
Oceanogr Mar Biol Ann Rev, 2001, 39: 103-164

10 Nakaoka M, Matsui S. Annual variation in the growth rate of Yoldia notabilis (Bivalvia: Nuculanidae) in Otsuchi Bay, northeastern Japan, analyzed using shell microgrowth patterns. Mar Bio, 1994, 119: 397-404

11 Schöne B R, Flessa D L, Dettman D H, et al. Upstream dams and downstream clams: Growth rates of bivalve mollusks unveil impact of river management on estuarine ecosystems (Colorado River Delta, Mexico). Estuar Coast Shelf S, 2003, 58: 715-726

12 Helama S, Schöne B R, Kirchhefer A J, et al. Compound response of marine and terrestrial ecosystems tovarying climate: Pre-anthropogenic perspective from bivalve shell growth increments and tree-rings. Mar Environ Res, 2007, 63: 185-199

13 Banni M, Bouraoui Z, Ghedira J, et al. Seasonal variation of oxidative stress biomarkers in clams Ruditapes decussatus sampled from Tunisian coastal areas. Environ Monit Assess, 2008, 155: 119-128

14 Kanazawa T, Sato S. Environmental and physiological controls on shell microgrowth pattern of Ruditapes philippinarum (Bivalvia: Veneridae) from Japan. J Mollusc Stud, 2008, 74: 89-95

15 Carroll M L, Johnson B J, Henkes G A, et al. Bivalves as indicators of environmental variation and potential anthropogenic impacts in the southern Barents Sea. Mar Pollut Bull, 2009, 59: 193-206

16 Sejr M K, Sand M K, Jensen K T, et al. Growth and production of Hiatella arctica (Bivalvia) in a high-Arctic fjord (Young Sound, Northeast Greenland). Mar Ecol Prog Ser, 2002, 244: 163-169

17 Norkko J, Norkko A, Thrush S F, et al. Detecting growth under environmental extremes: Spatial and temporal patterns in nucleic acid ratios in two Antarctic bivalves. J Exp Mar Biol Ecol, 2005, 326: 144-156

18 Schöne B R, Giere O. Growth increments and stable isotope variation in shells of the deep-sea hydrothermal vent bivalve mollusk Bathymodiolus brevior from the North Fiji Basin, Pacific Ocean. Deep Sea Res Part I, 2005, 52: 1896-1910

19 Hallmann N, Burchell M, Schöne B R, et al. High-resolution sclerochronological analysis of the bivalve mollusk Saxidomus gigantea from Alaska and British Columbia: Techniques for revealing environmental archives and archaeological seasonality. J Archaeol Sci, 2009, 36: 2353-2364

20 Richardson C A, Seed R, Naylor E. Use of internal growth band for measuring individual and population growth rates in Mytilus edulis from offshore production platforms. Mar Ecol Prog Ser, 1990, 66: 259-265

21 Abele D, Stroahl U, Brey T, et al. Imperceptible senescence: Ageing in the ocean quahog Arctica islandica. Free Radic Res, 2008, 42: 474-480

22 Nudelman F, Gotliv B A, Addadi L, et al. Mollusk shell formation: Mapping the distribution of organic matrix components underlying a single aragonitic tablet in nacre. J Struct Biol, 2006, 153: 176-187

23 Wise S. Microarchitecture and mode of formation of nacre (motherof-pearl) in pelecypods, gastropods and cephalopods. Eclogae Geol, 1970, 63: 775-797

24 Erben H K, Watabe, N. Crystal formation and growth in bivalve nacre. Nature, 1974, 248: 128-130

25 Checa A G, Rodríguez-Navarro A B, Esteban-Delgado F J. The nature and formation of calcitic columnar prismatic shell layers in pteriomorphian bivalves. Biomaterials, 2005, 26: 6404-6414

26 Checa A G, Rodríguez-Navarro A. Self-organisation of nacre in the shells of Pterioida (Bivalvia: Mollusca). Biomaterials, 2005, 26: 1071-1079

27 Sudo S, Fujikawa T, Nagakura T, et al. Structures of mollusc shell framework proteins. Nature, 1997, 387: 563-556

28 Fang Z, Feng Q, Chi Y, et al. Investigation of cell proliferation and differentiation in the mantle of Pinctada fucata (Bivalve, Mollusca). Mar Biol, 2008, 153: 745-754

29 House M R, Farrow G E. Daily growth banding in the shell of the cockle, Cardium edule. Nature, 1968, 219: 1384-1386

Open Access This article is distributed under the terms of the Creative Commons Attribution License which permits any use, distribution, and reproduction in any medium, provided the original author(s) and source are credited. 